\title{
Monocarboxylate transporter 1 (MCT1) in calf and sheep stomach
}

\author{
D. Kirat ${ }^{1}$, H. Inoue ${ }^{1}$, H. Iwano ${ }^{2}$, H. Yokota ${ }^{2}$ and S. Kato ${ }^{1,3}$ \\ School of Veterinary Medicine, Rakuno Gakuen University, \\ ${ }^{1}$ Department of Veterinary Physiology, \\ ${ }^{2}$ Veterinary Biochemistry \\ 582-1 Bunkyodai-Midorimachi, Ebetsu, Hokkaido 069-8501, Japan
}

\begin{abstract}
This study focused on detection of monocarboxylate transporter 1 (MCT1) in mucosal tissues from the four compartments of stomach in calves and sheep on both protein and mRNA levels using Western blotting and reverse transcriptase-polymerase chain reaction (RT-PCR), respectively. MCT1 was more expressed in the forestomach (rumen, reticulum, omasum) than in true stomach (abomasum) of both animals, and it was considerably more abundant in abomasum of adult sheep than of calves. The results suggest that MCT1 can be regulated by dietary changes and its physiological function is postulated to influence the transport of SCFA and their metabolites in ruminant stomach.
\end{abstract}

KEY WORDS: monocarboxylate transporter 1, stomach, calves, sheep, RT-PCR, Western blot

\section{INTRODUCTION}

Monocarboxylate transporter 1 (MCT1) belongs to the proton-linked monocarboxylate transporter family including 14 isoforms that have been so far identified in mammals, each having a different tissue distribution (Halestrap and Meredith, 2004). Studies on epithelial cells of the gastrointestinal tract in human and monogastric animals (Garcia et al., 1995; Ritzhaupt et al., 1998; Halestrap and Price, 1999; Orsengio et al., 1999) as well as on the sheep rumen (Müller et al., 2002) suggested that only MCT1 plays a major role in the transport of various monocarboxylates such as lactate and ketone bodies, whereas other MCT isoforms seem to be of little or no importance. However, little is known about the existence of MCT1 in all the compartments of the ruminant stomach. Therefore, the present study was planned to examine the presence of MCT1 in calf and sheep stomach on both mRNA and protein levels.

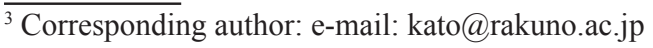




\section{MATERIAL AND METHODS}

\section{Animals and tissue samples}

A total of seven Holstein bull calves (5-14 days old) weighing 40-54 kg and three mature male Suffolk-strain sheep weighing 68-82 $\mathrm{kg}$ were used in this study. Calves were fed milk replacer until slaughtering, while sheep were fed hay $(100 \mathrm{~g})$ and lucerne pellets (2.5\% of body weight). Animals were euthanised by bleeding under anaesthesia with sodium pentobarbital. Mucosal samples from the four compartments of stomach (rumen, reticulum, omasum, and abomasum) were immediately collected from animals after slaughter and frozen in liquid nitrogen, and subsequently stored at $-80^{\circ} \mathrm{C}$ until use.

\section{Polyacrylamide gel electrophoresis (PAGE) and Western blot analysis}

Western blotting was performed as described previously (Garcia et al., 1995). Briefly, $30 \mu \mathrm{g}$ of membrane protein from the indicated tissue were separated on a $10 \%$ SDS-polyacrylamide gel and transferred to nitrocellulose membranes. The membranes were blocked overnight at $4^{\circ} \mathrm{C}$ with $5 \%(\mathrm{w} / \mathrm{v})$ nonfat dry milk in PBS$\mathrm{T}(0.1 \%$ Tween 20 in phosphate-buffered saline), then probed for $1 \mathrm{~h}$ with primary antibody (chicken anti-MCT1; AB1286; Chemicon International Inc., CA, USA) diluted 1:500 in PBS. After washing, the membranes were probed with rabbit antichicken IgY conjugated to HRP (Upstate, NY, USA) diluted 1:2000 in PBS and the signal detected by chemiluminescence (ECL, Amersham International, UK). Negative control blots were probed only with the secondary antibody.

\section{Reverse transcription-polymerase chain reaction ( $R T-P C R$ )}

Total RNA was isolated from mucosa of the collected tissues using TRIzol reagent according to the manufacturer's protocol (Life Technologies, GibcoBRL, Gaithersburg, MD, USA). MCT1-specific PCR primers were derived from ovine MCT1 partial cDNA sequence (GenBank accession no. AJ315929): sense primer 5'tggcatcttatcaggcagtgg-3'; antisense primer 5'-ccagccacacagcagtttaatag-3' (Hokkaido System Science Co., Ltd., Japan). Glyceraldehyde-3-phosphate dehydrogenase (GAPDH) was used as internal PCR control. PCR was performed using recombinant Taq DNA polymerase with a $55^{\circ} \mathrm{C}$ annealing temperature in a thermocycler $\left(\mathrm{iCycler}^{\mathrm{TM}}\right.$, Bio-Rad, USA) for 35 cycles. Nucleic acid sequence of inserts was determined using an automated DNA sequencer (310 Genetic Analyzer, ABI, Japan).

\section{RESULTS AND DISCUSSION}

Short chain fatty acids, SCFA (acetate, propionate and butyrate), the products of microbial fermentation of carbohydrates, are the primary energy source in ruminants (Bergman, 1990). Recently, it has been demonstrated that MCT1 may 
play a major role in the uptake of butyrate by the human and pig colonic luminal (membrane (Ritzhaupt et al., 1998). In this study we provided evidence of the expression of MCT1 in all compartments of ruminant stomach.

a

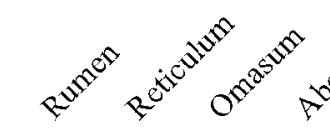

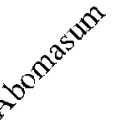

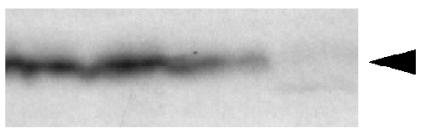

b

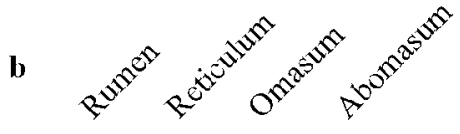

MCT1

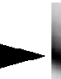

Figure 1. Western analysis of MCT1 protein content in membrane preparations from different parts of calf (a) and sheep (b) stomach

a

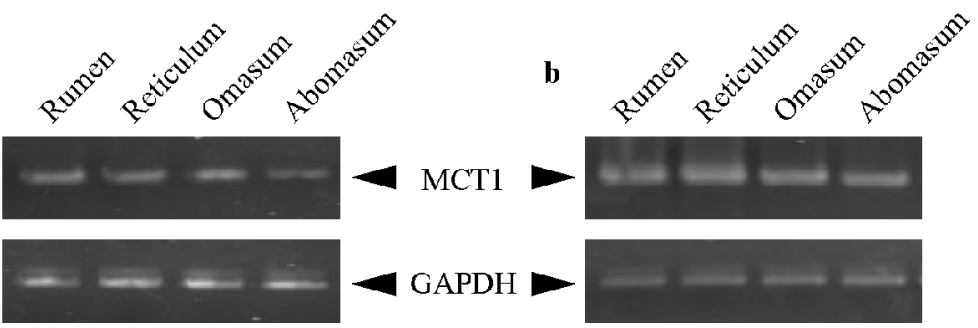

Figure 2. RT-PCR analysis of MCT1 from different parts of calf (a) and sheep (b) stomach

Using an antibody against MCT1, we identified a specific protein with an apparent molecular mass of 43-kDa on immunoblots of calf (Figure 1a) and sheep Figure 1b) stomach membranes. This band was not observed in the negative control blots (data not shown). The identified protein matches that of the $43-\mathrm{kDa}$ bands seen in blots of hamster tissues (Garcia et al., 1995). PCR products of the expected size (300 bp) were obtained by 35-cycle amplification of total RNA isolated from various parts of calf (Figure $2 a$ ) and sheep (Figure $2 b$ ) stomach. This band was observed after 30 cycles in all parts of stomach except in calf abomasum (data not shown). The high sequence homology with the analogous region of ovine and human MCT1 indicated the presence of mRNA encoding for MCT1 in all the examined samples. The results demonstrate that MCT1 is more expressed in the forestomach than in the abomasum of both animals which corresponds to the presence of higher level of SCFA in the forestomach. Interestingly, we found that MCT1 is considerably more abundant in abomasum of adult sheep than of milkfed calves, implying that the increased level of SCFA production in the stomach of adult sheep was able to up-regulate the expression of MCT1, suggesting that the expression and activity of MCT1 in ruminant stomach can be regulated by dietary changes. This is in agreement with Ferraris and Diamond (1989), who reported that the expression and activity of many nutrient transport proteins are adaptive 
to changes in their dietary substrate levels. The present study showed that the relative levels of MCT1 protein in calf and sheep stomach paralleled the pattern observed for the MCT1 mRNA. This suggests that MCT1 gene expression may be regulated at the transcriptional and/or post-transcriptional level.

During suckling of ruminant animals, the oesophageal groove closes, shunting liquid past the rumen into the abomasum. However, the oesophageal groove does not close completely, and some liquid may leak into the rumen (Church, 1969). This milk is fermented to SCFA (Lane et al., 2000). Under such condition, it is not surprising that MCT1 was expressed in the stomach of milk-fed calves.

\section{CONCLUSIONS}

MCT1 is expressed in the four compartments of calf and sheep stomach, and its physiological function is postulated to influence the transport of SCFA and their metabolites.

\section{REFERENCES}

Bergman E.N., 1990. Energy contributions of volatile fatty acids from the gastrointestinal tract in various species. Physiol. Rev. 70, 567-590

Church D.C., 1969. Digestive Physiology and Nutrition of Ruminants. Vol. 1. D.C. Church Publishing, Corvallis, OR

Ferraris R.P., Diamond J.M., 1989. Specific regulation of intestinal nutrient transporters by their dietary substrates. Ann. Rev. Physiol. 51, 125-141

Garcia C.K., Brown M.S., Pathak R.K., Goldstein J.L., 1995. cDNA cloning of MCT2, a second monocarboxylate transporter expressed in different cells than MCT1. J. Biol. Chem. 270, 1843-1849

Halestrap A.P., Price N.T., 1999. The proton-linked monocarboxylate transporter (MCT) family: structure, function, and regulation. Biochem. J. 343, 281-299

Halestrap A.P., Meredith D., 2004. The SLC16 gene family-from monocarboxylate transporters (MCTs) to aromatic amino acid transporters and beyond. Pflügers Arch. 447, 619-628

Lane M.A., Baldwin V.I., Jesse B.W., 2000. Sheep rumen metabolic development in response to age and dietary treatments. J. Anim. Sci. 78, 1990-1996

Müller F., Huber K., Pfannkuche H., Aschenbach J.R., Breves G., Gäbel G., 2002. Transport of ketone bodies and lactate in the sheep ruminal epithelium by monocarboxylate transporter 1 . Amer. J. Physiol. 283, G1139-G1146

Orsenigo M.N., Tosco M., Bazzini C., Laforenza U., Faelli A., 1999. A monocarboxylate transporter MCT1 is located at the basolateral pole of rat jejunum. Exp. Physiol. 84, 1033-1042

Ritzhaupt A., Wood I.S., Ellis A., Hosie K.B., Shirazi-Beechey S.P., 1998. Identification and characterization of a monocarboxylate transporter (MCT1) in pig and human colon: its potential to transport L-lactate as well as butyrate. J. Physiol. (London) 513, 719-732 